NABA TECHNICAL MEMORANDUM

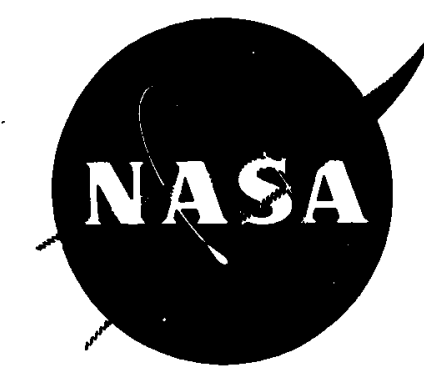

NASA TM $X-52182$

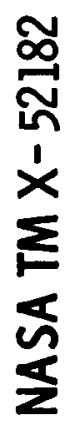

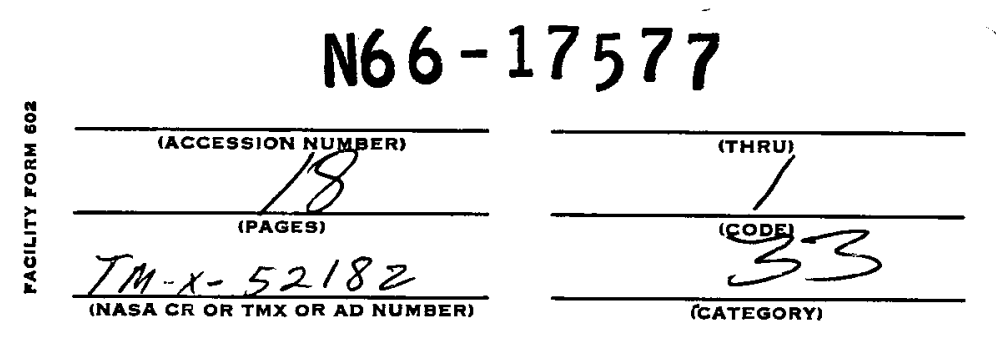

RAREFIED-GAS HEAT TRANSFER BETWEEN PARALLEL

PLATES BY A MONTE CARLO METHOD

by Morris Perlmutter

Lewis Research Center

Cleveland, Ohio
GPO PRICE $\mathbf{s}$

CFSTI PRICE(S) $\$$

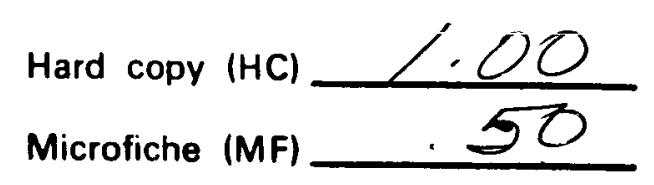

H 653 July 65

TECHNICAL PAPER proposed for presentation at Third International Heat Transfer Conference Chicago, Illinois, August 8-12, 1966 


\title{
RAREFIED-GAS HEAT TRANSFER BETWEEN PARALLEL PLATES BY A MONTE CARLO METHOD
}

\author{
by Morris Perlmutter \\ Lewis Research Center \\ Cleveland, Ohio
}

TECHNICAL PAPER proposed for presentation at

Third International Heat Transfer Conference

Chicago, Illinois, August 8-12, 1966 
RAREFIED-GAS HEAT TRANSFER BETWEEN PARALIEL PIATES BY A MONTE CARLO METHOD

\author{
by Morris Perlmutter \\ Lewis Research Center \\ National Aeronautics and Space Administration \\ Cleveland, Ohio
}

ABSTRACT

The problem of rarefied-gas heat transfer between parallel plates is treated by a Monte Carlo procedure. Hard-sphere molecule collisions are assumed. The distributions of target molecules are assumed to; be locally Maxwellian, with thermal velocity and density varying across the channel. The temperature, density, and heat transfer are found for flow conditions ranging from Knudsen numbers of 0.05 to the freemolecule regime for a wall-temperature ratio of 1 to 4 . The present results were compared with the results of another Monte Carlo solution obtained by using a different method and were found to agree with those results; they were also compered with other analytical solutions. The present results also agreed with the results obtained from a continuum solution with slip boundaries except that the continuum conductivity was too high for the larger Knudsen number cases.

AUSZUG

Die Frage der Wärmeübertragung in verdünntem Gas zwischen parallelen Platten wird mit Hilfe einer Monte-Carlo Methode behandelt. Zusammenstלsse von Hartsphärenmolekülen werden angenommen. Es wird vorausgesetzt dass die Beschussmoleküle örtlich eine Maxwellverteilung haben und dass die thermische Geschwindigkeit und Dichte im Querschnitt veränderlich sind. Die Methode liefert die Temperatur, Dichte und Wärmeübertragung für Strömungsbedingungen, die zwischen Knudsenzahlen von 0.05 und dem freien Molekülfluss für ein Verhältnis von Wand zu Temperatur bei $1: 4$ liegen. Die vorliegenden Ergebnisse werden mit den Resultaten einer anderen Monte-Carlolosung unter Verwendung einer unterschiedlichen Methode verglichen und stimmten mit diesen Resultaten überein; sie wurden auch noch mit anderen analytischen Lösungen verglichen. Die gegenwärtigen Resultate waren auch im Gleichklang mit durch eine Kontinuumlösung mit Gleitlinien erhaltenen Ergebnisse aber die Kontinuumleitfähigkeit war zu hoch in Fällen mit grösseren Knudsenzablen.

\title{
АННОТАЦИЯ
}

Исследуется теплопередача разреженным газом между паралельными пластинами способом Монте-Карло. Предпологается соударение жестких сферических молекул. Принлто местное максвелловское распределение молекул мишени с переменной скоростью теплового движения и переменной плотностью поперек канала. Температура, плотность и теплопередача найдены для режима обтеканния с числами Кнудсена начиная от 0.05 до режима обтекания со свободными молекулами при значениях коэффициента "стена-температура" от 1 до 4. Результаты были сравнены с результатами решения полученного другим способом Монте-Карло и оказались с ними в согласии. Результаты также были сравнены с другими аналитическими решениями. Они также в согласии с результатами полученными для сплошной среды с гранидами скольжения; но проводимость сплошной среды оказалась чрезмерно высокой для случаев с более высокими иислами Кнудсена.

\section{INTRODUCTION}

In rarefied-gas transport problems, the Navier-Stokes' equation for momentum transfer or the Fourier equation for conduction are no longer applicable because these equations assume local isotropy and smell gradients compared with the peth lengths of the molecules. Therefore, the more fundamental Boltzmann equation must be resorted to. This equation is difficult to treat by the usual analytical procedures because of its complexity.

Most of the analyses of rarefied-gas heat transfer have been for the linearized problem. As yet, few nonlinear results have been published. The present analysis of heat transfer between parallel plates at different temperatures enclosing a rarefied gas with hard-sphere molecule collisions 1s by a Monte Carlo procedure. This method reduces the complexity of the analysis thereby avoiding many of the simplifying assumptions generally made in the usual analytical procedures but at the expense of added numerical computations.
A linearized solution to the problem of heat transfer between parallel plates enclosing a hardsphere gas was analyzed by Gross and ziering [1]. 1 They assume the difference in wall temperature was small compared to the magnitude of the wall temperatures so that the problem can be described by the linearized Boltzmann equation. The distribution function was then approximated by half-range polynomials in velocity space and the space-dependent coefficient were determined by forming half-range moment equations.

The nonlinear problem was treated by Lavin and Haviland [2] for hard-sphere molecule collisions by using Lee's moment method. This method consists of choosing a distribution of molecular velocities in the form of half Maxwellians that contain a number of functions related to the local density and thermal velocity. A sufficient number of moment equations are then solved to determine these functions. Haviland and Lavin have also treated this problem by a Monte Carlo procedure

\footnotetext{
$1_{\text {Numbers }}$ in brackets denote references.
} 
[3], [4], and [5]. The major difference between the present analysis and that of Haviland and Lavin is in the methods of choosing targetmolecule collision partners and evaluating the local mean properties.

\section{The Monte Carlo Procedure}

The Monte Carlo procedure is a model sampling technique. A model is created and histories of sample molecules are followed through this model by making choices at points of decision from the appropriate probability distribution. By averaging certain properties of the sample molecules at various positions, macroscopic quantities of interest can be obtained.

The model is shown in Figure 1. The wall at $x_{2}$ of 0 is considered to be at temperature $\mathrm{T}_{\mathrm{w}, 0}$ and the other wall at $\mathrm{x}_{2}$ of $\mathrm{D}$ is at temperature $T_{\mathrm{W}, 1}$. A rarefied, hard-sphere molecule gas is contained between the walls.

The sample molecule history is started at wall 0 by picking its velocity components from the appropriate distribution of velocities of the absorbed and reemitted molecules. The space between the walls is divided into zones as shown in Figure 1. The sample molecule, after leaving the wall, will either pass through the first zone or will have a collision with a target molecule in this zone. This will depend on whether the path length to collision for the sample molecule is longer or shorter than the distance the sample molecule must travel to pass through the zone. If the sample molecule passes through the first zone with no collision, it is started again at the beginning of the next zone with its velocity components unchanged.

If there is a collision in the zone, however, the point of collision is found at the end of the path length to collision. The distribution of target-molecule velocities is assumed to have the form of a Maxwellian distribution throughout each zone. The distribution of target-molecule collision partners is derived from this distribution of target molecules. A target molecule is picked from the distribution of target-molecule collision partners in the zone. A collision calculation is carried out for the sample- and target-molecule collision partners, and new velocity components are found for the sample molecules after collision. The history of the sample molecule is then continued from the point of collision with the new sample-molecule velocity components. The collision partner is ignored after collision for reasons discussed in [4].

A new path length to collision is found for the sample molecule, and this is compared in length with the distance from the point of collision to the zone boundary. If the peth length to collision is greater, the sample molecule is started at the beginning of the next zone with its velocity components unchanged. In the other case, there is a collision in the zone and the collision calculations are repeated as before.

If the sample molecule strikes the wall at $\mathrm{x}_{2}=\mathrm{D}$, it is absorbed and reemitted with new velocity components picked from the appropriate distribution of velocities based on the temperature of the wall. After leaving the upper wall it is followed as before.

The transport and fluid characteristics of interest are density distribution, temperature distribution, and heat transfer across the channel.
These can be obtained [6] by locating scoring positions at various locations across the channel (see Fig. 1). By scoring the various characteristics of the sample molecules as they pass the scoring cross section, the transport properties and fluid characteristics of interest can be obtained at these scoring positions.

In the present analysis it was assumed that the target molecules in each zone were in a Maxwellian velocity distribution based on the local temperature and density of each zone. From the Monte Carlo solution based on this assumed distribution of target molecules, the temperature and density at each scoring position are found. The Monte Carlo solution is then rerun with the new local temperature and density in the Maxwellian distribution of target molecules. The problem was iterated in this manner until the density and temperature distribution found from the sample molecule histories agreed with the density and temperature distribution used for the target molecules.

In [3], [4], and [5], a Monte Carlo solution is used to treat the present problem. That solution is carried out with an assumed distribution of target molecules in a tabular form. By scoring the velocity components of many sample molecules as they pass through each zone, the velocity distribution of the molecules in each zone can be obtained in tabular form. These velocity distributions are then used as the target-molecule distributions in the next iteration. This process is continued until the sample-molecule distribution found agrees with the target-molecule distribution assumed. Then, from the distributions in each zone, the macroscopic quantities of interest can be found by numerical integration of the moments of the distribution. This was carried out for one hardsphere-molecule case with a Knudsen number of 2 and a wall-temperature ratio of 1 to 4 . In the present method, it is not necessary to find and store the entire distributions of target moleculess in each increment since the form of the target molecule distribution is assumed.

In the present Monte Carlo solution, since the collision partners are ignored, only conservation of mass is satisfied exactly since molecules are not allowed to "disappear" in transit between the walls. Conservation of momentum and energy will only be satisfied if the correct target molecule distribution is assumed.

\section{ANALYSIS}

Velocity Components of Emitted Sample Molecule

The sample molecule history begins at the surface at temperature $T_{W}, 0$. It is assumed that the molecules incident on the surface are perfectly accommodated; that is, they are in a Maxwellian distribution at the wall, based on the wall temperature. This assumption is discussed in [7]. In this case, the normalized Maxwellian velocity distribution of the molecules moving away from the wall $V_{2}>0$ is given as

$\frac{\rho_{+, 0}}{M} f_{+, 0} \mathrm{~d}^{3} \mathrm{v}=\frac{2 \rho_{+, 0}}{\mathrm{Mr}^{3 / 2} \mathrm{C}_{\mathrm{w}, 0}^{3}}\left[\exp \left(-\frac{\mathrm{v}^{2}}{\mathrm{C}_{\mathrm{w}, 0}^{2}}\right)\right] d \mathrm{v}_{1} \mathrm{dv}_{2} \mathrm{dv}_{3} \mid \begin{aligned} & \text { (1) } \\ & \mathrm{v}_{2}>0\end{aligned}$

The $\rho_{+}, 0 / M$ is the number density of molecules moving away from the wall, and $C_{w, 0}$ is the ther- 
mal velocity based on the wall temperature $\left[(2 \mathrm{k} / \mathrm{M}) \mathrm{T}_{\mathrm{W}, \mathrm{O}}\right]^{1 / 2}$.

If we consider the positive $x_{2}$ direction normal to the wall, the distribution of velocities of the molecules leaving the wall per unit time per unit area is $\mathrm{V}_{2} f_{+, O}([4]$ and [6]). This distribution can be transformed to cylindrical coordinates $V_{1}=V_{r} \cos \Theta, v_{2}=v_{2}, v_{3}=v_{r} \sin \theta$ and can be normalized by $\left\langle V_{2}\right\rangle_{+, 0}=C_{w}, 0 / \sqrt{\pi} \quad$ [4]
The $V_{2}, V_{r}$, and $\theta$ picked give the direction and velocity of the sample molecule as it leaves the wall. This same result was used in [4] and [7]. Finally, $V_{1}$ and $V_{3}$ can be obtained from

$\mathrm{V}_{3}=\mathrm{V}_{\mathrm{r}} \sin \Theta ; \quad \mathrm{V}_{1}=\mathrm{V}_{\mathrm{r}} \cos \Theta$

$$
f\left(\Theta, v_{2}, v_{r}\right)=\frac{v_{2} f+0^{a^{3} v}}{\left\langle v_{2}\right\rangle_{+, 0}}=\frac{2 v_{2}}{\pi C_{w, 0}^{4}}\left[\exp \left(-\frac{v^{2}}{C_{w, 0}^{2}}\right)\right] v_{r} d \Theta d v_{2} d v_{r}=f_{\Theta} f_{v_{2}} f_{v_{r}} d \Theta d v_{2} d v_{r}
$$

The distributions for $f_{\Theta}^{f} V_{2} f V_{r}$ can be written separately as

$$
\begin{gathered}
f_{\Theta} d \Theta=\frac{d \Theta}{2 \pi} \\
f_{v_{2}} d v_{2}=\frac{2 v_{2}}{c_{\mathrm{w}, 0}^{2}}\left[\exp \left(-\frac{v_{2}^{2}}{c_{\mathrm{w}, 0}^{2}}\right)\right] d v_{2} \\
f_{v_{r}} d v_{R}=\frac{2 v_{r}}{c_{\mathrm{w}, 0}^{2}}\left[\exp \left(-\frac{v_{r}^{2}}{c_{\mathrm{w}, 0}^{2}}\right)\right] d v_{r}
\end{gathered}
$$

The velocity components of our sample molecule leaving the surface must be picked from these distributions. A convenient way of picking from a distribution for the high-speed computer is to transform the distribution to a uniform distribu$t i o n$ in $R$ by setting the random number $R$ equal to the cumlative distribution function. For example,

$$
R=\int_{0}^{\Theta} f_{\Theta} d \Theta=\frac{\Theta}{2 \pi}
$$

Then, by using a high-speed computer to generate a random number $R$ between 0 and 1 , $\Theta$ can be obtained from Equation (4) such that for a large number of samples picked in this manner, the aistribution in tquation ( $3 a$ ) will be satisfied.

Similarly, $v_{2}$ can be picked from

$$
\mathrm{R}_{2}=\int_{0}^{\mathrm{v}_{2}} \frac{2 \mathrm{v}_{2}}{\mathrm{c}_{\mathrm{w}, 0}^{2}}\left[\exp \left(-\frac{\mathrm{v}_{2}^{2}}{\mathrm{C}_{\mathrm{w}, 0}^{2}}\right)\right] \mathrm{dv}_{2}
$$

Or, since picking $\mathrm{R}$ is equivalent to picking $1-\mathrm{R}$,

$$
v_{2}=\left(-c_{w, 0}^{2} \ln R_{V_{2}}\right)^{1 / 2}
$$

so that by picking $\mathrm{R}_{2} \mathrm{~V}_{2}$ can be obtained from Equation (6).

The velocity $V_{r}$ is obtained in the same manner and is given by

$$
\mathrm{v}_{\mathrm{r}}=\left(-\mathrm{C}_{\mathrm{w}, 0}^{2} \ln \mathrm{R}_{\mathrm{v}_{\mathrm{r}}}\right)^{\mathrm{I} / 2}
$$

\section{Calculation of the Path Iength $\lambda$ to Collision}

The probability that a sample molecule will collide in the incremental path length $\lambda$ to $\lambda+d \lambda$ is given in [8] as

$$
f_{\lambda}=\frac{\exp \left(-\lambda / \lambda_{S}\right)}{\lambda_{S}}
$$

where $\lambda_{S}$ is the mean free path to collision of the sample molecule moving at velocity $V_{S}$ in that zone.

A path length to collision for the sample molecule from this distribution can then be chosen by the same procedure described earlier.

$$
\lambda=-\lambda_{S} \ln R_{\lambda}
$$

To use this relation, the mean free path $\lambda_{5}$ in the zone must first be calculated.

As shown in Appendix A (Eq. (AI4)), the mean free path of a sample molecule moving at velocity $V_{S}$ through a Maxwellian gas at density $\rho$ with a thermel velocity $C$ is

$$
\frac{\lambda_{S}}{D}=\frac{2^{1 / 2} K n \mu_{s}}{\frac{\rho}{\rho_{A}}\left[\frac{\exp \left(-\mu_{s}^{2}\right)}{\pi^{1 / 2}}+\left(\operatorname{erf} \mu_{s}\right)\left(\mu_{s}+\frac{1}{2 \mu_{s}}\right)\right]}
$$

where $\mu_{s}$ is the velocity of the sample molecule nondimensionalized by the thermal velocity of the zone $\left(\mu_{S}=V_{S} / C\right)$. Equation (10) can then be used in Equation $(9)$ to obtain the distance to collision for the sample molecule in the zone.

\section{Calculating New Sample-Molecule Velocity Components After Collision}

The distribution of velocities of the target molecules colliding with the sample molecule is given in Appendix $B$ follows the derivation in [8]. From this distribution, the velocity components of the target-molecule collision partner as given by Equation (B12) are chosen. The new velocity components of the sample molecule after collision with the target-molecule collision partner are given, following the derivation in [4]. 


$$
\begin{aligned}
& v_{1 s}^{*}=\frac{1}{2}\left(v_{1 s}+v_{1 t}\right)+v_{R}\left(1-b^{2}\right)^{1 / 2} H \\
& v_{2 s}^{*}=\frac{1}{2}\left(v_{2 s}+v_{2 t}\right)+\frac{1}{2} v_{R}\left(1-2 b^{2}\right) \\
& v_{3 s}^{*}=\frac{1}{2}\left(v_{3 s}+v_{3 t}\right)+v_{R}\left(1-b^{2}\right)^{1 / 2} \Xi
\end{aligned}
$$

where

$$
\begin{aligned}
v_{R}=\left[\left(v_{1 s}-v_{1 t}\right)^{2}+\left(v_{2 s}\right.\right. & \left.-v_{2 t}\right)^{2} \\
& \left.+\left(v_{3 s}-v_{3 t}\right)^{2}\right]^{1 / 2}
\end{aligned}
$$

The values of $H$ and $\Xi$ are obtained by picking two random numbers that are used in the following equations

$$
\mathrm{H}=2 \mathrm{R}_{\mathrm{H}}-1 \quad \Xi=2 \mathrm{R}_{\Xi}-1 \quad \text { and } \quad \mathrm{b}^{2}=\mathrm{H}^{2}+\Xi^{2}
$$

where $b^{2}$ must be less than 1 . If $b^{2}$ is greater than 1 , a new set of random numbers must be chosen to find $H$ and $\Xi$

\section{Macroscopic Flow Properties}

The macroscopic fluid characteristics needed are density, temperature, and heat transfer across the channel. Scoring positions are located at various distances across the channel; as shown in Figure 1. The average quantity of $Q$ transported across the scoring cross section $p$ in the positive $x_{2}$ direction, can be written as

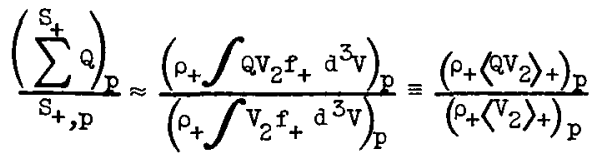

where $S_{+} p$ is the number of sample molecules passing across the scoring cross section $p$ in the positive $x_{2}$ direction and $Q$ is some quantity that each sample molecule carries. Similarly, the average quantity $Q$ transported in the negative $\bar{x}_{2}$ uirection is

$$
\frac{\left(\sum_{-, p}^{S} Q\right)_{p}}{S_{-}} \approx \frac{\left(\rho_{-}\left\langle Q V_{2}\right\rangle-\right)_{p}}{\left(\rho_{-}\left(V_{2}\right\rangle-\right)_{p}}
$$

Since there is no net flow across the channel

$$
S_{+, p}=S_{-, p}
$$

and

$$
\left(\rho_{+} \int \nabla_{2^{f}+} d^{3} V\right)_{p}=-\left(\rho_{-} \int \nabla_{2^{f}}-d^{3} V\right)_{p}
$$

Hence,

$$
\frac{\rho\left\langle v_{2} Q\right\rangle_{p}}{\rho_{+, p}\left\langle v_{2}\right\rangle_{+, p}}=\frac{1}{s_{+, p}}\left(\sum^{S_{+}} Q-\sum^{-} Q\right)_{p}
$$

The number of sample molecules that pass the scoring cross section at $p$ in the positive $V_{2}$ direction, $s_{+, p}$, divided by the number of times the sample molecule leaves wall $0, N$ can be related to the mass flux passing in the positive $V_{2}$ direction at $p$ by

$$
\frac{s_{+, p}}{N} \approx \frac{\rho_{+, p}\langle V\rangle_{+, p}}{\rho_{+, 0}\langle V\rangle_{+, 0}}
$$

where $\rho_{+}, 0\langle\nabla\rangle_{+}, 0$, the mass flux leaving wall 0 , is equal to $p_{+}, O_{w, O} / \pi^{1 / 2}$ [4]. Combining Equations (16) and (17) results in

$$
\rho\left\langle V_{2} Q\right\rangle_{p}=\frac{\rho_{+,} O_{W, O} C}{N_{\pi} I / 2}\left(\sum_{+}^{S_{+}} Q-\sum_{-}^{S_{-}} Q\right)_{p}
$$

If $Q$ is taken as $1 / v_{2}$, Equation (18) becomes

$$
\frac{\rho}{p_{+, 0}}=\frac{c_{w, 0}}{N \pi^{1 / 2}}\left(\sum^{s_{+}} \frac{1}{v_{2}}-\sum_{-\frac{1}{v_{2}}}^{s_{-}}\right)
$$

The average density in the channel is then obtained by averaging the density of all the scoring cross sections from $p=0$ to $p=p$.

$\frac{\rho_{A}}{\rho_{+, 0}}=\frac{1}{p_{f}}\left[\frac{1}{2}\left(\frac{\rho_{0}}{\rho_{+, 0}}+\frac{\rho_{p_{f}}}{\rho_{+, 0}}\right)+\sum_{p=1}^{p_{f}-1} \frac{\rho_{p}}{\rho_{+, 0}}\right]$

As shown in [4], the continuum solution, $\mathrm{Kn} \rightarrow 0$, is given by

$$
\frac{\rho}{\rho_{A}}=\frac{1+r_{T}^{3 / 2}+r_{T}}{3\left[1-\left(1-r_{T}^{3 / 2}\right)_{X}\right]^{2 / 3}}
$$

where $\mathrm{r}_{\mathrm{T}}=\mathrm{T}_{\mathrm{w}, 1} / \mathrm{T}_{\mathrm{w}, 0}$

The temperature can be obtained in a similar manner. The undirected kinetic energy per unit mass of a monatomic gas is equal to $1 / 2(\mathrm{kT} / \mathrm{M})$ times the degrees of freedom

$$
\frac{3}{2} \frac{k}{M} T=\frac{1}{2}\left\langle V^{2}\right\rangle
$$

From Equation (18), this can be written as

$$
\frac{T}{T_{w, 0}}=\frac{2}{3\left(\frac{\rho}{\rho_{+, 0}}\right) N \pi^{1 / 2} C_{w, 0}}\left[\sum\left(\frac{v^{2}}{V_{2}}\right)-\sum\left(\frac{v^{2}}{v_{2}}\right)\right]_{p}
$$

where $\rho /\left(\rho_{+}, 0\right)$ is given by Equation (19). As shown in $[4]$, the collisionless solution, $\mathrm{Kn} \rightarrow \infty$, is given by

$$
\left(\frac{T}{T_{W}, 0}\right)_{K n \rightarrow \infty}=r_{T}^{1 / 2}
$$

and the continuum solution, $\mathrm{Kn} \rightarrow 0$, is given by 


$$
\frac{T}{T_{w}, 0}=\left[1-\left(1-r_{T}^{3 / 2}\right) x\right]^{2 / 3}
$$

Finaliy, the heat transer across the channel can be obtained from

$$
\begin{aligned}
q & =\frac{\rho}{2}\left\langle v_{2}\left(v_{2}^{2}+v_{r}^{2}\right)\right\rangle \\
& =\frac{\rho_{+}, o_{w} c_{, 0}}{2 \pi^{1 / 2_{N}}}\left[\sum^{S_{+}, p}\left(v_{2}^{2}+v_{r}^{2}\right)-\sum^{S_{-} p}\left(v_{2}^{2}+v_{r}^{2}\right)\right]
\end{aligned}
$$

This can be nondimensionalized by $\frac{1}{2}\left[\rho\left\langle v_{2}\left(v_{2}^{2}+v_{r}^{2}\right)\right\rangle\right]_{+, 0}=\frac{1}{\pi^{1 / 2}}\left(\rho_{+}, 0 c_{w}^{3}, 0\right)$, and then, by using the $\rho_{+} / \rho_{A}$ previously obtained, the following is found:

$$
\begin{aligned}
& \frac{\pi^{1 / 2}}{\rho_{A} c_{w, 0}^{3}} q=\frac{1}{2 N c_{w, 0}^{2}}\left[\sum^{s_{+} p}\left(v_{2}^{2}+v_{r}^{2}\right)\right. \\
& \left.-\sum^{\mathrm{s}, \mathrm{p}}\left(\mathrm{v}_{2}^{2}+\mathrm{v}_{\mathrm{r}}^{2}\right)\right]\left(\frac{\rho_{+, 0}}{\rho_{\mathrm{A}}}\right)
\end{aligned}
$$

The heat transfer is then averaged across the channel as in the case of the density (Eq. (2OD)). The heat transfer is compared to the collisionless result as derived in [4]:

$$
\left(\frac{\pi^{I / 2} q}{\rho_{A} c_{w}^{3}}\right)_{K n \rightarrow \infty}=r_{T}^{1 / 2}-r_{T}
$$

and to the continuum solution as given by

$$
\left(\frac{\pi^{2} / 2 q}{\rho_{A} c_{\mathrm{w}}^{3}, 0}\right)_{K n \rightarrow 0}=\frac{25}{64} \pi\left(1-r_{T}^{3 / 2}\right)_{K n}
$$

\section{RESULTS AND CONCLUSIONS}

The temperature snd density distribution results are shown in Figure 2 for a Knudsen number of 2 and wall-temperature ratios of 0.25 and 0.75 . These results are compared with the one case carried out by the Monte Carlo procedure of [4] and are in good agreement. The nonlinear moment solution results [2] have a significantly different slope than the Monte Carlo results.

The linearized solution [1] is also compared with the present results. For the smaller walltemperature difference case $\left(T_{\mathrm{w}}, I / T_{\mathrm{w}}, 0=0.75\right)$, the results agree with the linearized solution. For the larger wall-temperature differences, however, where $T_{w, 1} / T_{\mathrm{w}, 0}=0.25$, the temperature is different in magnitude from the present result, although the shapes of the curve agree. In the linearized solution the centerline temperature is a parameter taken midway between the wall temperatures. The present results have their centerline temperature significantly below this value for the Knudsen number of 2 ; hence the large disagreement between the results.
Also shown in Figure 2 are the slip continuum results. The continuum equations are solved by using the fluid temperature (slip temperature) near the walls rather than the wall temperatures as the boundary temperature. By using the slip temperatures that give the best fit to both the temperature and density results from the present solution, the results shown are obtained. These results indicate that the slip continuum densities and temperature curves agree well with the present results. The present results show the magnitude of the temperature slip between the wall and the fluid near the wall as well as the Knudsen layer where the fluid temperature near the wall deviates from the continuum results toward the wall temperature.

In Figure 3, the temperature and density results for a range of Knudsen numbers are shown. These results are for a fixed value walltemperature ratio of 0.25 . They agree with the collisionless solution for the very large Knudsen number and approach the continuum solution for the values of the smaller Knudsen number. Figure 3 also shows the temperature and density distribution obtained from the aforementioned continuum slip solutions.

In Figure 4, the heat fiux nondimensionalized by the continuum heat flux is plotted as a function of the inverse Knudsen number for the walltemperature ratio of 0.25 . The Monte Carlo result of [4] was solved for the inverse Knudsen number of 0.5 and found to agree with the present results and with the linearized solution of [1]. The nonlinear solution gave results above the linear solution, while the present results rell below the linear solution for small knudsen numbers.

Also shown in Figure 4 is the heat transfer from the continuum solution with the slip boundary conditions. The slip boundary heat transfer is very high for larger Knudsen numbers, so that the slip approximation does not apply near the collisionless region. This could be because the noncontinuum conductivity, which is the first term in the series expansion of the second approximation in the Enskog method [4], is not appropriate in the present noncontinuum case. The results of Figure 4 indicate that if a conductivity lower than the continuum conductivity is used in the continuum solution with slip, the results would be in good agreement with the present case. In conclusion, the present result agrees with the results obtained by the Monte Carlo method used in [4] for the one case that wos carried out $\left(\mathrm{Kn}=2, \mathrm{~T}_{\mathrm{w}, 1} / \mathrm{T}_{\mathrm{w}}, 0=0.25\right)$. The linear and the nonlinear solutions disagreed somewhat with the present result. This could be due partly to the approximation made in the other analysis and partly to the present assumption that the target molecules locally are in a Maxwellian distribution. The assumption of a local Mexwellian distribution for the target molecules would be most applicable near equilibrium conditions, that is, cases with small temperature gradients and short mean free paths. The present results are being extended to the more realistic two-sided Maxwellian as was used in [2].

The results indicate the continuum solution with slip boundaries, if the slip can be properly predicted, would give good results, except that for the larger Knudsen numbers, the continuum conductivity value is too high.

The Monte Carlo method seems to give reasonable results for this problem. There are certain drawbacks to the method, however. The error is 
reduced by running a large number of samples, which run into large amounts of computing time. The computing time needed for a sample molecule was found to increase exponentially as the Knudsen number approached zero. On the IBM 7094, typical sample run times for 10,000 samples were: $K n=5$, 5 minutes; $\mathrm{Kn}=2,6$ minutes; $\mathrm{Kn}=0.5,10 \mathrm{~min}$ utes; $\mathrm{Kn}=0.2,15$ minutes; $\mathrm{Kn}=0.1,25$ minutes; and $\mathrm{Kn}=0.05,45$ minutes. However, for problems of the present type, which are difficult to solve by other methods without over simplifying assumptions, the present method proved very useful.

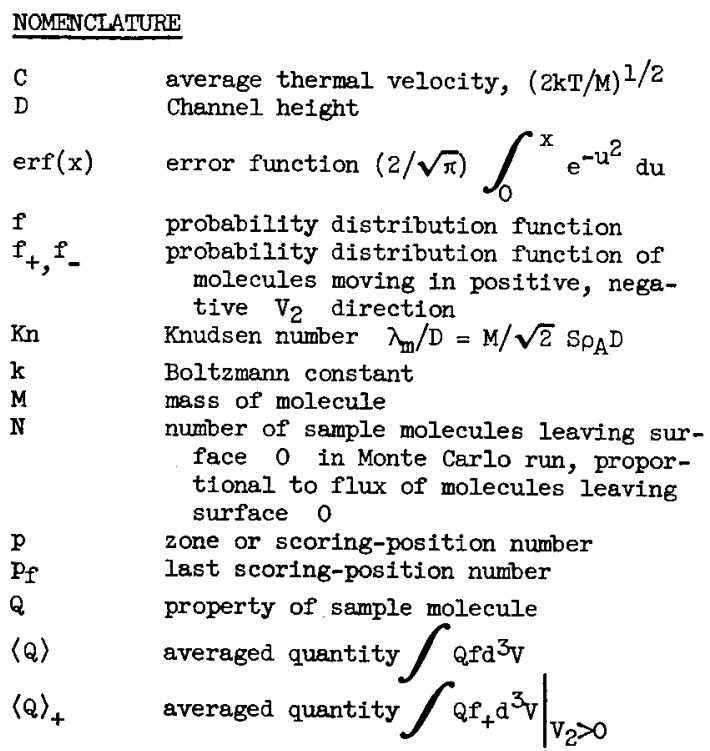
averaged quantity $\mathrm{Qfd}^{3} \mathrm{~V}$ averaged quantity $\left.\int \mathrm{Qf}_{+} \mathrm{d}^{3} \mathrm{~V}\right|_{\mathrm{V}_{2}>0}$

random number between 0 and $I$ wall temperature ratio $\mathrm{T}_{\mathrm{w}, 1} / \mathrm{T}_{\mathrm{w}, 0}$ mutual collision cross section $\pi \sigma^{2}$ number of sample molecules through scoring position in positive, negative $x_{2}$ direction

absolute temperature molecular velocity radial velocity; $\left(v_{1}^{2}+v_{3}^{2}\right)^{1 / 2}$

velocity after collision coordinates

defined by Eqs. (B6) and (B7) defined by Eqs. (B6) and (B7) collision rate of sample molecule with target molecules

path length to collision

mean free path length

dimensionless velocity, $\mathrm{V} / \mathrm{C}$ defined by Eq. (AII)

mass density

diameter of hard-sphere molecule angle between sample-molecule and target-molecule velocities

Subscripts :

$\begin{array}{ll}\mathrm{A} & \text { averaged } \\ \mathrm{p} & \text { increment number } \\ \mathrm{p}_{\mathrm{f}} & \text { last increment } \\ \mathrm{R} & \text { relative velocity } \\ \mathrm{s} & \text { sample molecule } \\ \mathrm{t} & \text { target molecule } \\ \mathrm{w}, 0 ; \mathrm{w}, 1 & \text { at wall } 0,1 \\ 0,1 & \text { evaluated next to wall } 0,1 \\ 1,2,3 & \text { coordinate directions } \\ +,- & \text { positive or negative direction }\end{array}$

\section{APPENDIX A}

EVALUATION OF MEAN FREE PATH

The number of collisions per unit time $d \Theta$ of a sample molecule moving at velocity $V_{S}$ through target molecules in velocity volume space $\mathrm{d}^{3} \mathrm{v}_{t}$ for hard-sphere molecules is given in [8] as

$$
d \Theta=\rho f_{t} V_{R} S d^{3} v_{t}
$$

where $V_{R}$ is the velocity of the target molecules relative to the sample-molecule velocity before collision, $v_{R, i}=v_{t, i}-v_{s, i}$, and $s$ is the mutul-collision cross section $\pi \sigma^{2}$ where $\sigma$ is the diameter of the molecule. The relative velocity $\nabla_{R}$ can then be written as

$$
\begin{aligned}
v_{R}=\left[\left(v_{1 t}-v_{1 s}\right)^{2}+\left(v_{2 t}-v_{2 s}\right)^{2}\right. & \\
& \left.+\left(v_{3 t}-v_{3 s}\right)^{2}\right]^{1 / 2}
\end{aligned}
$$

For a Maxwellian distribution of target molecules, the collision rate can be written as

$$
\begin{array}{r}
d \Theta=\frac{\rho S}{N_{G}^{3} / 2 c_{p}^{3}}\left(\exp -\frac{v_{1 t}^{2}+v_{2 t}^{2}+v_{3 t}^{2}}{c_{p}^{2}}\right) \\
\quad \times \nabla_{R} d v_{1 t} d v_{2 t} D_{3 t}
\end{array}
$$

Then transforming variable by a rotation, using Eulerian angles as given in [9], yields

$$
v_{i t}=A_{i j}^{-1} \nabla_{j t}^{\prime}
$$

where

$$
A_{i j}^{-1}=\left|\begin{array}{ccc}
\frac{v_{2 s}}{v_{r s}} & \frac{v_{1 s}}{v_{s}} & -\frac{v_{3 s} v_{1 s}}{v_{r s} v_{s}} \\
-\frac{v_{1 s}}{v_{r s}} & \frac{v_{2 s}}{v_{s}} & -\frac{v_{3 s} v_{2 s}}{v_{r s} v_{s}} \\
0 & \frac{v_{3 s}}{v_{s}} & \frac{v_{r s}}{v_{s}}
\end{array}\right|
$$


where the inverse transformation is

$$
v_{i t}^{\prime}=A_{i j} v_{j t}
$$

where

$$
A_{i j}=\left|\begin{array}{ccc}
\frac{v_{2 s}}{v_{r s}} & -\frac{v_{1 s}}{v_{r s}} & 0 \\
\frac{v_{1 s}}{v_{s}} & \frac{v_{2 s}}{v_{s}} & \frac{v_{3 s}}{v_{s}} \\
-\frac{v_{3 s} v_{1 s}}{v_{r s} v_{s}} & -\frac{v_{2 s} v_{3 s}}{v_{r s} v_{s}} & \frac{v_{r s}}{v_{s}}
\end{array}\right|
$$

(A5b)

and where

$v_{r}=\left(v_{I}^{2}+v_{2}^{2}\right)^{1 / 2}$ and $v=\left(v_{r}^{2}+v_{3}^{2}\right)^{1 / 2}$

$(A 6 a)$

Then, the following is obtained:

$d \Theta=\frac{\rho S}{M \pi^{3 / 2} c_{p}^{3}}\left(\exp -\frac{v_{1 t}^{\prime 2}+v_{2 t}^{\prime 2}+v_{3 t}^{\prime 2}}{c_{p}^{2}}\right)$

$\times\left[v_{1 t}^{\prime 2}+\left(v_{2 t}^{\prime}-v_{s}\right)^{2}+v_{3 t}^{\prime 2}\right]^{1 / 2} d v_{1 t}^{\prime} d v_{2 t}^{\prime} d v_{3 t}^{\prime}$

By transforming into spherical coordinates

$$
\begin{aligned}
& v_{1 t}^{\prime}=v_{t} \cos \theta \sin \varphi \\
& v_{2 t}^{\prime}=v_{t} \cos \varphi \\
& v_{3 t}^{\prime}=v_{t} \sin \theta \sin \varphi
\end{aligned}
$$

The following is obtained:

$\mathrm{d} \Theta=\frac{\rho S}{M \pi^{3 / 2} C_{p}^{3}}\left(\exp -\frac{v_{t}^{2}}{C_{p}^{2}}\right) v_{R} v_{t}^{2} \sin \varphi d \varphi d \theta d v_{t}$

where

$$
\bar{v}_{R}=\left(\bar{v}_{t}^{2}+\bar{v}_{s}^{2}-\bar{z}_{s} \bar{v}_{t} \cos \psi\right)^{l / 2}
$$

This can be written as

$d \Theta=\frac{C_{p} \rho S}{M_{\pi}{ }^{3 / 2}}\left[\exp \left(-\mu_{t}^{2}\right)\right] \mu_{t}^{2} \mu_{R} \sin \phi d \varphi d \theta d \mu_{t}$ where $\mu$ is the nondimensionalized velocity

$\mathrm{v} / \mathrm{c}$. To obtain the total collision rate for the target molecules over all velocities, Equation (A9) must be integrated over $\varphi, \theta$, and $\mu_{t}$. This can be integrated over $\theta$ from 0 to $2 \pi$ and over $\varphi$ from 0 to $\pi$ to give

$$
\mathrm{d} \Theta\left(\mu_{t}\right)=\frac{4 \rho C_{p} S}{M \pi^{1 / 2}}\left[\exp \left(-\mu_{t}^{2}\right)\right] \mu_{t}^{2} \mu_{R, A} d \mu_{t}
$$

where

$u_{R, A}=\int_{0}^{\pi}\left(\mu_{t}^{2}+\mu_{S}^{2}-2 \mu_{S} \mu_{t} \cos \varphi\right)^{1 / 2} \frac{\sin \varphi}{2} d \varphi$

or

$$
\mu_{R, A}=\left\{\begin{array}{l}
\mu_{s}+\mu_{t}^{2} / 3 \mu_{s} \text { if } \mu_{s}>\mu_{t} \\
\mu_{t}+\mu_{s}^{2} / 3 \mu_{t} \text { if } \mu_{t}>\mu_{s}
\end{array}\right.
$$

Integrating over $\mu_{t}$ from 0 to $\infty$ to obtain the total collision frequency for a sample molecule moving at velocity $\mu_{s}$ through a Maxwellian gas will then produce

$$
\theta=\frac{\rho S C_{p}}{M}\left[\frac{\exp \left(-\mu_{S}^{2}\right)}{\pi / 2}+\left(\operatorname{erf} \mu_{S}\right)\left(\mu_{s}+\frac{1}{2 \mu_{S}}\right)\right]
$$

The nondimensional mean free path is then found, as discussed in [8], by dividing the samplemolecule velocity by the total collision rate to give

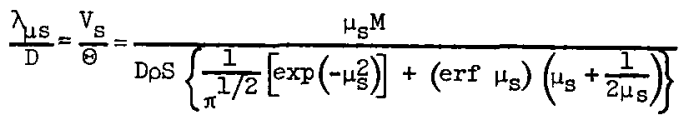

By using the difinition of Knudsen number given by Equation (11) the following can be written:

$$
\begin{aligned}
\frac{\lambda \mu_{S}}{D}=K n 2^{I / 2} \mu_{s}\left(\frac{\rho}{p_{A}}\right)^{-1}\left\{\frac{\left[\exp \left(-\mu_{S}^{2}\right)\right]}{\pi^{I / 2}}+\right. \\
\left.+\left(\operatorname{erf} \mu_{S}\right)\left(\mu_{S}+\frac{I}{2 \mu_{s}}\right)\right\}
\end{aligned}
$$

APPENDTX B

PICKING THE TARGET-MOLECULE COLLISION PARTNER

The velocity distribution of target molecules that the sample molecule will collide with as discussed in [1] is given by

$$
\frac{d \theta}{\theta}=\frac{\left[\exp \left(-\mu_{t}^{2}\right)\right] \mu_{t}^{2} \mu_{R} \sin \phi d \varphi d \theta d \mu_{t}}{\pi\left[\exp \left(-\mu_{S}^{2}\right)\right]+\pi / 2\left(\operatorname{erf} \mu_{S}\right)\left(\mu_{S}+\frac{1}{2 \mu_{S}}\right)}
$$

The distribution in $\theta$ for the target molecules is readily seen to be

$$
f_{\theta} d \theta=\frac{d \theta}{2 \pi}
$$

Then, the $\theta$ can be picked from this distribution by 


$$
\theta=2 \pi R_{\theta}
$$

The distribution of $\mu_{t}$ for the target molecules is obtained from the marginal distribution

$\mathbf{f}_{\mu t} \alpha_{\mu t}=\frac{4\left[\exp \left(-\mu_{t}^{2}\right)\right] \underset{\mu_{t}, \mu_{R, A}}{2} d_{\mu_{t}}}{\left[\exp \left(-\mu_{S}^{2}\right)\right]+\pi^{1 / 2}\left(\operatorname{erf} \mu_{S}\right)\left(\mu_{S}+\frac{1}{2 \mu_{S}}\right)}$

We can then pick from this distribution as before

$\mathrm{R}_{\mu_{t}}=\frac{\gamma\left(\mu_{s}, \mu_{t}\right)+\eta\left(\mu_{s}, \mu_{t}\right)}{\left[\exp \left(-\mu_{s}^{2}\right)\right]+\pi^{1 / 2}\left(\operatorname{erf} \mu_{s}\right)\left(\mu_{s}+\frac{1}{2 \mu_{s}}\right)}$

where, when $\mu_{\mathrm{s}}>\mu_{\mathrm{t}}$,

$$
\begin{aligned}
& r\left(\mu_{s}, \mu_{t}\right)=-2 \mu_{s} \mu_{t}\left[\exp \left(-\mu_{t}^{2}\right)\right]+\mu_{s} \sqrt{\pi}\left(\operatorname{erf} \mu_{t}\right) \\
& -\frac{2}{3} \frac{\mu_{t}^{3}}{\mu_{s}}\left[\exp \left(-\mu_{t}^{2}\right)\right]-\frac{\mu_{t}}{\mu_{s}}\left[\exp \left(-\mu_{t}^{2}\right)\right]+\frac{\sqrt{\pi}}{2 \mu_{s}}\left(\operatorname{erf} \mu_{t}\right)
\end{aligned}
$$

and

$$
\eta=0
$$

and, when $\mu_{s}<\mu_{t}$,

$$
r\left(\mu_{s}, \mu_{t}\right)=r\left(\mu_{s}, \mu_{s}\right)
$$

and

$$
\left.\begin{array}{rl}
\eta=-2[ & \left.\exp \left(-\mu_{t}^{2}\right)\right]\left(\frac{\mu_{s}^{2}}{3}+1+\mu_{t}^{2}\right) \\
+ & {\left[2 \exp \left(-\mu_{s}^{2}\right)\right]\left(\frac{4 \mu_{s}^{2}}{3}+1\right)}
\end{array}\right\}
$$

To find $\phi$ for the target molecule, the distribution of target molecules as a product of a marginal distribution times a conditional distribution can be written:

$$
\frac{d \Theta\left(\mu_{t}, \varphi\right)}{\Theta}=f\left(\mu_{t}\right) f\left(\varphi \mid \mu_{t}\right)
$$

Then, $\varphi$ can be picked from the conditional distribution

$f\left(\varphi \mid \mu_{t}\right)=\frac{\left(\mu_{t}^{2}+\mu_{s}^{2}-2 \mu_{s} \mu_{t} \cos \phi\right)^{1 / 2} \frac{\sin \phi d \varphi}{2}}{\mu_{R, A}}$

Picking $\varphi$ for a given $\mu_{t}$ is found as before from

$R_{\varphi}=\frac{\left(\mu_{t}+\mu_{s}-2 \mu_{t} \mu_{s} \cos \varphi\right)^{3 / 2}-\left|\mu_{t}-\mu_{s}\right|^{3}}{\left(\mu_{t}^{2}+\mu_{s}^{2}+2 \mu_{t} \mu_{s}\right)^{3 / 2}-\left|\mu_{t}-\mu_{s}\right|^{3}}$

$$
\begin{aligned}
V^{\prime} 1 t & =\mu_{t} C_{p} \cos \theta \sin \varphi \\
V^{\prime} 2 t & =\mu_{t} C_{p} \cos \varphi \\
V_{3 t}^{\prime} & =\mu_{t} C_{p} \sin \theta \sin \varphi
\end{aligned}
$$

and from Equation (A4)

$$
\begin{aligned}
& v_{1 t}=V^{\prime} 1 t\left(\frac{V_{2 s}}{V_{r s}}\right)+V^{\prime} 2 t\left(\frac{v_{1 s}}{V_{s}}\right)-V^{\prime} 3 t\left(\frac{v_{3 s} V_{1 s}}{V_{r s} V_{s}}\right) \\
& v_{2 t}=-v^{\prime} 1 t\left(\frac{v_{1 s}}{V_{r s}}\right)+V^{\prime} 2 t\left(\frac{v_{2 s}}{v_{s}}\right)-V^{\prime} 3 t\left(\frac{v_{2 s} v_{3 s}}{V_{r s} v_{s}}\right) \\
& v_{3 t}=0+V^{\prime} 2 t\left(\frac{V_{3 s}}{V_{s}}\right)+V^{\prime} 3 t\left(\frac{V_{r s}}{V_{s}}\right)
\end{aligned}
$$

\section{REFERENCES}

1. Gross, E. P. ; and S. Ziering: "Heat Flow Between Parallel Plates," Phys. Fluids, vol. 2, No. 6, November-December, 1959, pp. 701-712.

2. Lavin, M. L. ; and J. K. Haviland: "Application of a Moment Method to Heat Transfer in Rarefied Gases," Phys. Fluids, vol. 5, No. 3, March, 1962, pp. 274-279.

3. Lavin, M. L.: "A Monte Carlo Solution for Heat Transfer in Rarefied Gases," Massachusetts Inst. of Tech. Rep. No. 61-3, AFOSR Rept. No. 627, May, 1961.

4. Haviland, J. K.: "Monte Carlo Application to Molecular Flows," Massachusetts Inst. of Tech., Rep. No. 61-5, AFCRL Scientific Rep. 648, May, 1961.

5. Haviland, J. K.; and M. L. Lavin: "Application of the Monte Carlo Method to Heat Transfer in a Rarefied Gas," Phys. Fluids, vol. 5, No. 11, November, 1962, pp. 1399-1405.

6. Perlmutter, M. : "Monte Carlo Solution for the Characteristics of a Highly Ionized Gas Flowing Through a Channel with a Transverse Magnetic Fleld," NASA TN D-2211, October, 1964: See also vol. 2 of Fourth Symposium on Rarefied Gas Dynamics, J. H. deLeeuw, ed., Academic Press, New York, to be published 1966.

7. Patterson, G. N.: "A state-ố-tine-Airt Survey of Some Aspects of the Mechanics of Rarefied Gases and Plasmas," Toronto University, AFARL 64-60, DDC No. AD-600873, April, 1964.

8. Kennard, E. H.: Kinetic Theory of Gases with and Introduction to Statistical Mechanics, McGraw-Hill Book Co., Inc., New York, 1938.

9. Goldstein, H.: Classical Mechanics, AddisonWesley Press, Cambridge, Massachusetts, 1950. are obtained from 


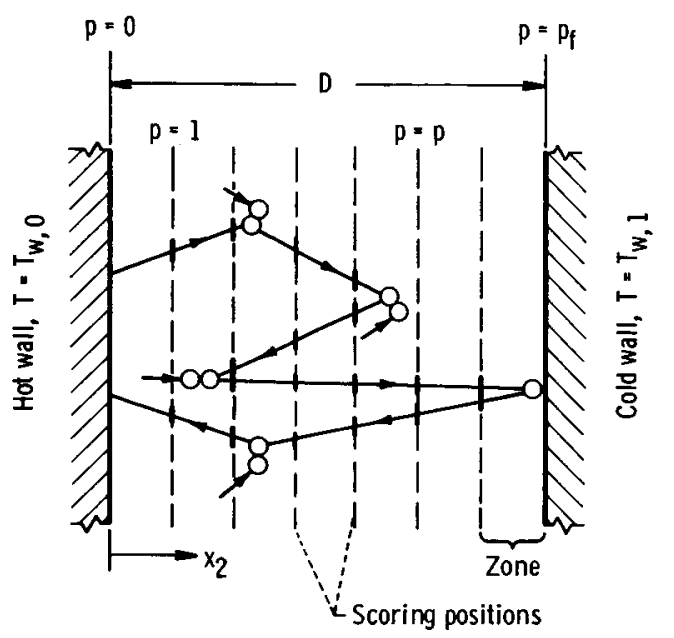

Figure 1. - Model. 

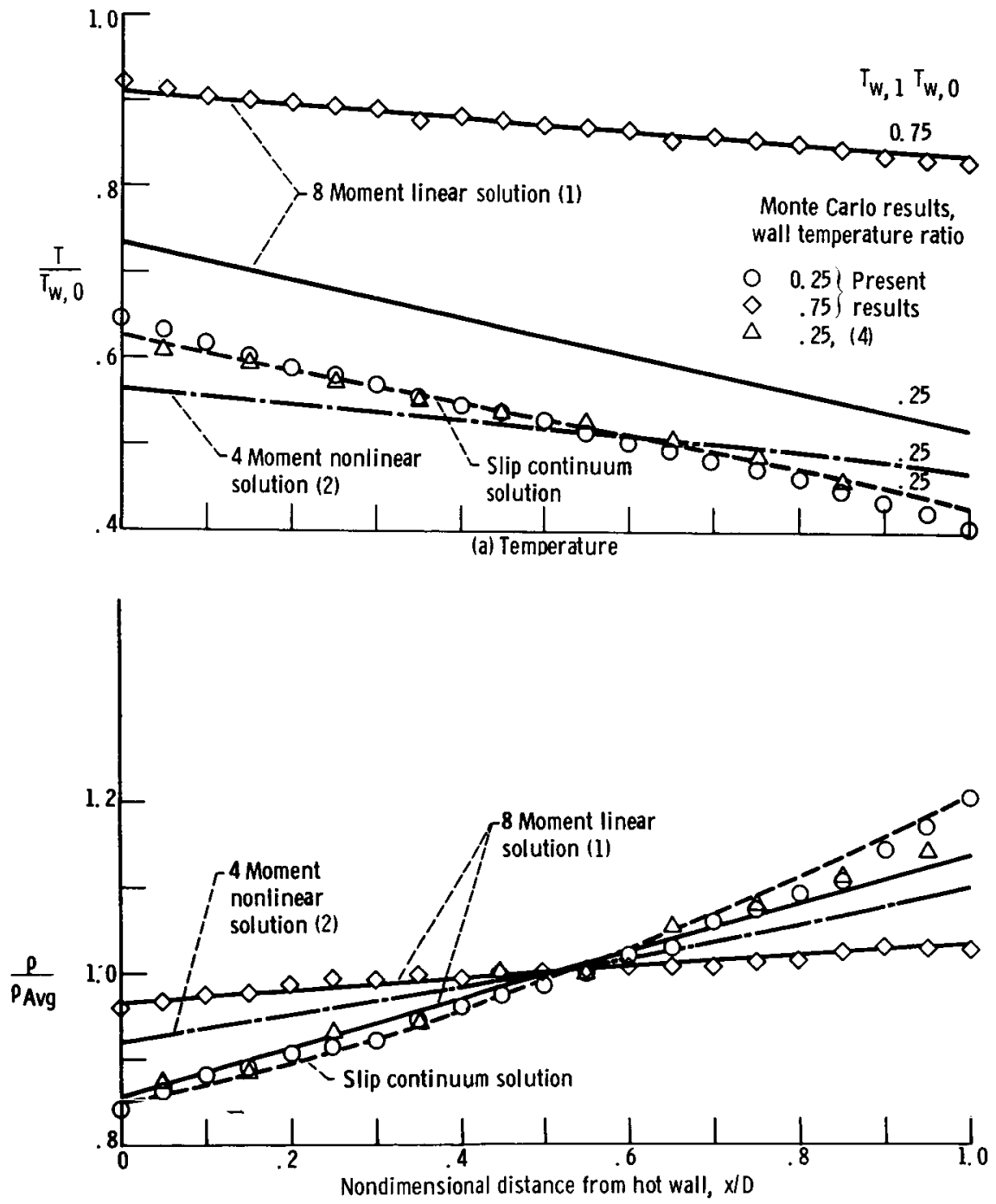

(b) Density

Figure 2. - Distributions across channel for Knudsen number 2. 

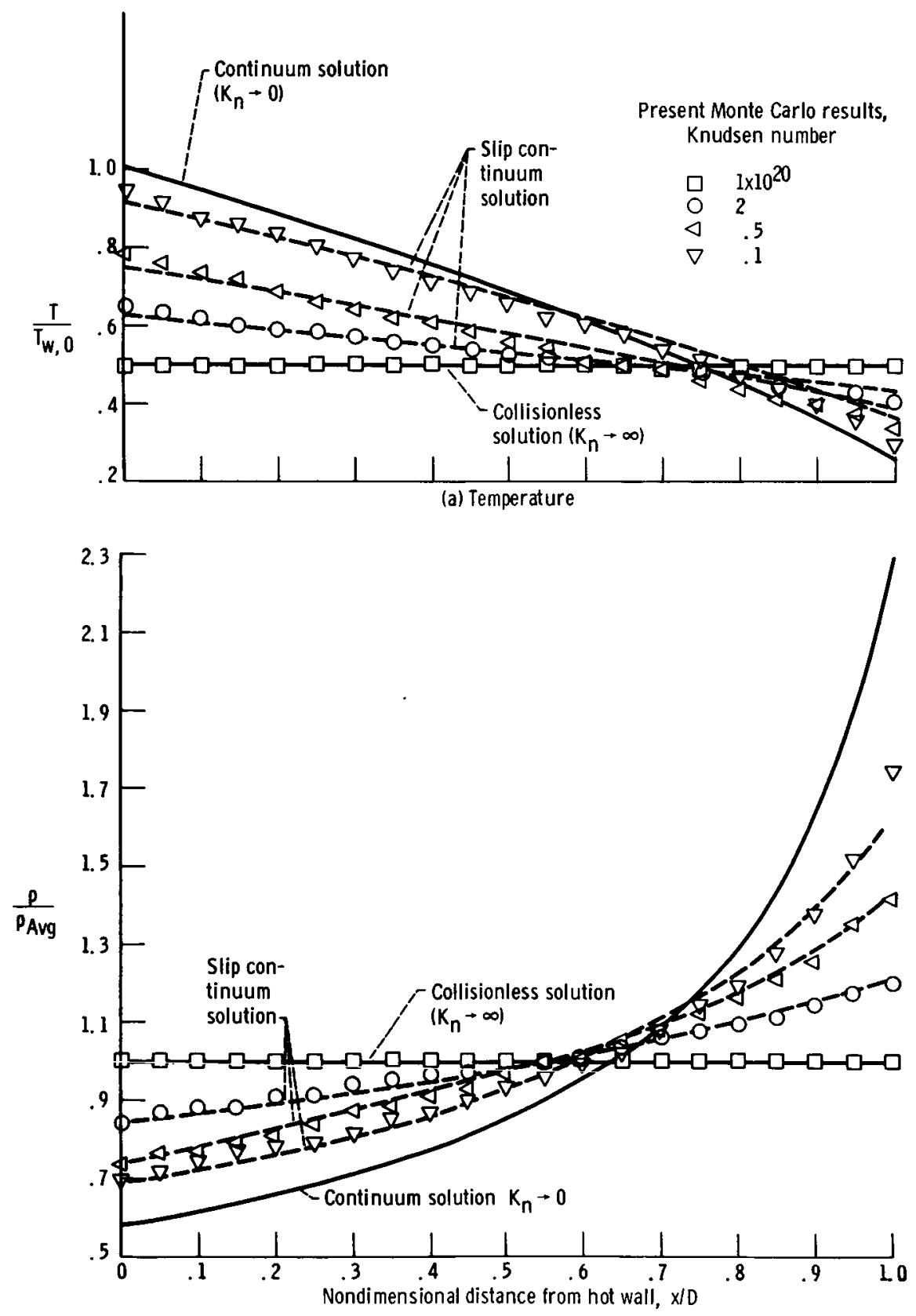

(b) Density

Figure 3. - Distributions for various Knudsen numbers; wall temperature ratio, 0.25. 


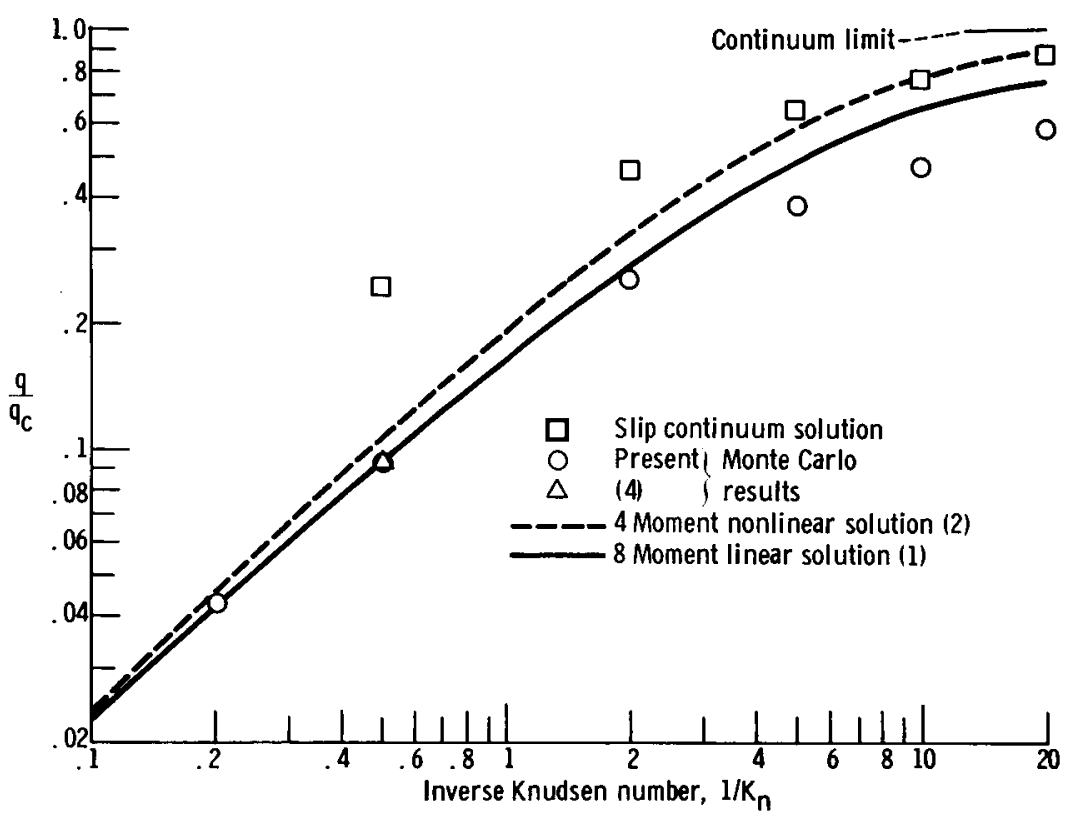

Figure 4. - Dimensionless heat flux for wall-temperature ratio of 0.25. 